JOURNAL OF

SYMPLECTIC GEOMETRY

Volume 2, Number 2, 267-278, 2004

\title{
EQUIVARIANT SYMPLECTIC HODGE THEORY AND THE $d_{G} \delta$-LEMMA
}

\author{
Yi LiN AND REYER SJAMAAR
}

\begin{abstract}
Consider a Hamiltonian action of a compact Lie group on a symplectic manifold which has the strong Lefschetz property. We establish an equivariant version of the MerkulovGuillemin $d \delta$-lemma and an improved version of the KirwanGinzburg equivariant formality theorem, which says that every cohomology class has a canonical equivariant extension.
\end{abstract}

\section{Introduction}

Adapting a familiar notion in Riemannian Hodge theory Brylinski [Bry88] introduced the symplectic Hodge star operator and defined a differential form $\alpha$ on a symplectic manifold to be harmonic if $d \alpha=d * \alpha=0$. He conjectured that on a compact symplectic manifold every de Rham cohomology class contains a harmonic representative and proved this conjecture to be true for Kähler manifolds and certain other examples.

A compact symplectic manifold $(M, \omega)$ of dimension $2 n$ is said to have the strong Lefschetz property if the map

$$
H^{n-k}(M) \longrightarrow H^{n+k}(M), \quad c \longmapsto[\omega]^{k} \wedge c
$$

is an isomorphism for each $0 \leq k \leq n$. Mathieu [Mat95] proved the remarkable theorem that Brylinski's conjecture is true for $M$ if and only if it has the strong Lefschetz property. This result was sharpened by Merkulov [Mer98] and Guillemin [Gui01], who independently established the symplectic $d \delta$-lemma. Let $\delta= \pm * d *$ be Koszul's boundary operator and suppose $M$ has the strong Lefschetz property. The $d \delta$-lemma asserts that if $\alpha$ is a harmonic $k$-form on $M$ that is either exact or coexact then $\alpha=d \delta \beta$ for some $k$-form $\beta$.

Mathematics Subject Classification. 53D20, 58A12. 
The object of this paper is to extend these results to an equivariant setting. Let $M$ be a compact symplectic manifold satisfying the strong Lefschetz condition and let $G$ be a compact connected Lie group acting on $M$ in a Hamiltonian fashion. One of our main results is an equivariant version of the $d \delta$-lemma, namely the $d_{G} \delta$-lemma, which is obtained by replacing $d$ with the equivariant exterior derivative $d_{G}$ on the Cartan double complex $\Omega_{G}(M)=\left(S \mathfrak{g}^{*} \otimes \Omega(M)\right)^{G}$.

It was proved by Kirwan [Kir84] and Ginzburg [Gin87] that the spectral sequence of the Cartan complex associated to the horizontal filtration degenerates at the first term. This implies the equivariant formality theorem, which states that the equivariant cohomology group $H_{G}^{*}(M)$ is isomorphic to $\left(S \mathfrak{g}^{*}\right)^{G} \otimes H^{*}(M)$ as a vector space. This isomorphism depends on a choice of an equivariantly closed extension for each closed form on $M$. The $d \delta$-lemma produces a very simple proof of the Kirwan-Ginzburg theorem, which replaces the Morse theory in [Kir84, Gin87] with Hodge theory and which shows in addition that there is a canonical choice for these equivariantly closed extensions up to coboundaries and therefore a canonical isomorphism from $H_{G}^{*}(M)$ to $\left(S \mathfrak{g}^{*}\right)^{G} \otimes H^{*}(M)$. The idea behind this result, which appears to be new even for Kähler manifolds, goes back to Deligne [Del68], who showed how Lefschetz type properties lead to the collapse of spectral sequences.

In view of the Kirwan surjectivity theorem these results suggest a close relationship between the harmonic theory of $M$ and that of its symplectic quotients. This will be the topic of a future publication.

Section 2 of this paper is a brief review of symplectic Hodge theory, including the $d \delta$-lemma. In Section 3 we deduce from the $d \delta$-lemma the strong version of the equivariant formality theorem and the $d_{G} \delta$-lemma.

\section{Symplectic Hodge theory}

Let $(V, \omega)$ be a $2 n$-dimensional symplectic vector space. The symplectic form induces an isomorphism $b: V \rightarrow V^{*}$ defined by $\left\langle a^{b}, b\right\rangle=\omega(a, b)$, where $\langle\cdot, \cdot\rangle$ denotes the dual pairing between $V$ and $V^{*}$. Let $\sharp: V^{*} \rightarrow V$ be the inverse of $b$. Then $\omega(v, w)=\omega\left(v^{\sharp}, w^{\sharp}\right)$ is a symplectic form on $V^{*}$, which induces bilinear pairings on $\Lambda V^{*}$ also denoted by $\omega$ and defined by

$$
\omega\left(v_{1} \wedge \cdots \wedge v_{k}, w_{1} \wedge \cdots \wedge w_{k}\right)=\operatorname{det} \omega\left(v_{i}, w_{j}\right)
$$

for $v_{i}, w_{j} \in V^{*}$. It is easy to show that $\omega$ is nondegenerate; furthermore on $\Lambda^{k} V^{*}$ it is anti-symmetric when $k$ is odd and symmetric when $k$ is even. For $a \in V$ let $\iota(a): \Lambda^{k} V^{*} \rightarrow \Lambda^{k-1} V^{*}$ denote inner product by $a$ and for $u \in V^{*}$ let $\varepsilon(u): \Lambda^{k} V^{*} \rightarrow \Lambda^{k+1} V^{*}$ denote exterior product by $u$. An easy calculation confirms the following fact. 
Lemma 2.1. For all $u \in V^{*}$ the maps $\varepsilon(u)$ and $-\iota\left(u^{\sharp}\right)$ are adjoint with respect to $\omega$.

The symplectic Hodge star operator $*: \Lambda^{k} V^{*} \rightarrow \Lambda^{2 n-k} V^{*}$ is defined by

$$
u \wedge * v=\omega(u, v) \frac{\omega^{n}}{n !}
$$

for $u, v \in \Lambda^{k} V^{*}$.

Example 2.2. A direct calculation shows that $* \omega / k !=\omega^{n-k} /(n-k)$ ! for all $k$. In short, $* \exp \omega=\exp \omega$.

The Hodge star operator has the following properties.

Lemma 2.3 ([Bry88]). (i) Let $\left(V_{1}, \omega_{1}\right)$ and $\left(V_{2}, \omega_{2}\right)$ be symplectic vector spaces and let $(V, \omega)$ be their direct sum. Denote the Hodge star operators on $V_{1}, V_{2}$ and $V$ by $*_{1}, *_{2}$ and $*$, respectively. Then

$$
*\left(u_{1} \wedge u_{2}\right)=(-1)^{k_{1} k_{2}}\left(*_{1} u_{1}\right) \wedge\left(*_{2} u_{2}\right)=\left(*_{2} u_{2}\right) \wedge\left(*_{1} u_{1}\right)
$$

for all $u_{1} \in \Lambda^{k_{1}} V_{1}^{*}$ and $u_{2} \in \Lambda^{k_{2}} V_{2}^{*}$.

(ii) Let $(V, \omega)$ be a symplectic vector space. Then $*(* u)=u$ for all $u \in$ $\Lambda^{k} V^{*}$, i.e. $*^{2}=\mathrm{id}$.

Now let $(M, \omega)$ be a $2 n$-dimensional compact symplectic manifold. Applying the above construction to each tangent space we obtain a symplectic Hodge star operator $*: \Omega^{k}(M) \rightarrow \Omega^{2 n-k}(M)$ satisfying

$$
\alpha \wedge * \beta=\omega(\alpha, \beta) \frac{\omega^{n}}{n !}
$$

for $\alpha, \beta \in \Omega^{k}(M)$. The symplectic boundary operator $\delta: \Omega^{k} \rightarrow \Omega^{k-1}$ is then defined by

$$
\delta \alpha=(-1)^{k+1} * d * \alpha
$$

for $\alpha \in \Omega^{k}(M)$. As in Riemannian Hodge theory we call $\alpha$ coclosed if $\delta \alpha=0$, coexact if $\alpha=\delta \beta$ for some $\beta$, and harmonic if it is closed and coclosed. However, in a striking departure from the Riemannian case, the symplectic Laplacian $d \delta+\delta d$ vanishes identically, i.e. $d$ and $\delta$ anti-commute.

Example 2.4. Every function is coclosed. A function $f$ is coexact if and only if the $2 n$-form $* f=f * 1=f \omega^{n} / n$ ! is exact, which is the case if and only if $\int_{M} f \omega^{n} / n !=0$, i.e. the mean of $f$ relative to the Liouville measure is zero.

Let $\pi=\omega^{\sharp}$ be the Poisson two-vector defined by the symplectic form and let $v_{f}=(d f)^{\sharp}$ denote the Hamiltonian vector field of a function $f$. The first part of the following result, which shows that $\delta$ can be defined on any Poisson manifold, is Koszul's original definition of $\delta$ (see [Kos85]) and the second part is a Leibniz rule for $\delta$. 
Proposition 2.5. $\quad$ (i) $\delta=[\iota(\pi), d]$.

(ii) $\delta(f \alpha)=f \delta \alpha-\iota\left(v_{f}\right) \alpha$ for all functions $f$ and forms $\alpha$.

(iii) If $\alpha$ is closed, then $\delta \alpha$ is exact. If $\alpha$ is coclosed, then $d \alpha$ and $\iota\left(v_{f}\right) \alpha$ are coexact.

Proof. (i) is due to [Bry88, Theorem 2.2.1]. Let $\varepsilon(f)$ denote multiplication by $f$. Using (i) and the ordinary Leibniz rule $[d, \varepsilon(f)]=\varepsilon(d f)$ we get

$$
\begin{aligned}
{[\delta, \varepsilon(f)]=[[\iota(\pi), d], \varepsilon(f)]=[\iota(\pi),[d, \varepsilon(f)]]-[d,[\iota(\pi), \varepsilon(f)]] } & \\
= & {[\iota(\pi), \varepsilon(d f)] . }
\end{aligned}
$$

The identity $d f=\iota\left(v_{f}\right) \omega$ yields $\varepsilon(d f)=\left[\iota\left(v_{f}\right), \varepsilon(\omega)\right]$, so

$$
\begin{gathered}
{[\iota(\pi), \varepsilon(d f)]=\left[\iota(\pi),\left[\iota\left(v_{f}\right), \varepsilon(\omega)\right]\right]=\left[\iota\left(v_{f}\right),[\iota(\pi), \varepsilon(\omega)]\right]+\left[\left[\iota(\pi), \iota\left(v_{f}\right)\right], \varepsilon(\omega)\right]} \\
=\left[\iota\left(v_{f}\right), A\right]=-\iota\left(v_{f}\right),
\end{gathered}
$$

where we used $[\iota(\pi), \varepsilon(\omega)]=A$, the scalar operator defined by $A \alpha=(n-k) \alpha$ for $\alpha$ of degree $k$. Together with (1) this shows $[\delta, \varepsilon(f)]=-\iota\left(v_{f}\right)$, which is equivalent to (ii). The first statement in (iii) follows immediately from (i). (See [Bry88, Proposition 1.3.2].) Applying Hodge star we get that $d \alpha$ is coexact for $\alpha$ coclosed. Finally, if $\delta \alpha=0$, then $\iota\left(v_{f}\right) \alpha=-\delta(f \alpha)$ by (ii).

The following result of Mathieu can be thought of as an analogue of the Hodge theorem. (See also [Yan96] for an alternative proof.) Let $H(M)=$ $H(\Omega(M))$ denote the de Rham cohomology of $M$.

Theorem 2.6 ([Mat95]). The following conditions are equivalent.

(i) $M$ satisfies the strong Lefschetz condition, i.e. the map

$$
H^{n-k}(M) \longrightarrow H^{n+k}(M), \quad c \longmapsto[\omega]^{k} \wedge c,
$$

is an isomorphism for each $0 \leq k \leq n$.

(ii) Every cohomology class in $H(M)$ has a harmonic representative.

Let $\Omega_{\delta}(M)$ be the kernel of $\delta$. Since $d$ and $\delta$ anti-commute, $\Omega_{\delta}(M)$ forms a subcomplex of $\Omega(M)$, the cohomology of which we denote by $H_{\delta}(M)$. In this language Mathieu's result can be restated as follows.

Theorem 2.7. The strong Lefschetz property holds for $(M, \omega)$ if and only if the map $H_{\delta}(M) \rightarrow H(M)$ induced by the inclusion $\Omega_{\delta}(M) \hookrightarrow \Omega(M)$ is surjective.

This restatement is useful for the following reason. The identity

$$
\operatorname{ker} d \cap \operatorname{im} \delta=\operatorname{im} d \delta=\operatorname{ker} \delta \cap \operatorname{im} d
$$

is known as the $d \delta$-lemma. In plain language: if a $k$-form $\alpha$ is harmonic and either exact or coexact, then $\alpha=d \delta \beta$ for some $k$-form $\beta$. (In particular $\alpha$ is both exact and coexact.) If the $d \delta$-lemma holds for $M$, then for every closed form $\gamma$ the equation $\delta \gamma=d \delta \zeta$ is soluble for $\zeta$ and so the cohomology class 
of $\gamma$ has a harmonic representative. Therefore $M$ satisfies strong Lefschetz by Theorem 2.7. Intriguingly, the converse is also true.

Theorem 2.8 ([Mer98, Gui01]). Strong Lefschetz holds if and only if the

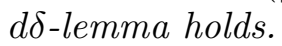

The following result is an easy consequence. Here $H(\Omega(M), \delta)$ denotes the homology of $\Omega(M)$ with respect to $\delta$.

Theorem 2.9 ([Mer98, Gui01]). Assume strong Lefschetz. Then the dchain maps in the diagram

$$
\Omega(M) \longleftarrow \Omega_{\delta}(M) \longrightarrow H(\Omega(M), \delta)
$$

are quasi-isomorphisms, i.e. they induce isomorphisms in cohomology. Thus the de Rham complex of $M$ is formal, i.e. quasi-isomorphic to a complex with zero differential.

This immediately implies the following sharpened version of Theorem 2.7.

Theorem 2.10 ([Gui01]). Strong Lefschetz holds if and only if the map $H_{\delta}(M) \rightarrow H(M)$ is bijective.

\section{Equivariant formality and the $d_{G} \delta$-lemma}

In this section we establish equivariant versions of Theorems 2.8-2.9. Let $G$ be a connected compact Lie group acting in a Hamiltonian fashion on a $2 n$-dimensional compact symplectic manifold $(M, \omega)$. Let $\phi: \mathfrak{g} \rightarrow C^{\infty}(M)$ be an equivariant moment map. Throughout this section we assume $(M, \omega)$ to have the strong Lefschetz property. (It is well-known that the Lefschetz and Hamiltonian conditions are not independent. For instance, under the Lefschetz assumption every symplectic $G$-action whose generating vector fields have fixed points is Hamiltonian.)

We start with a quick review of equivariant de Rham theory. See e.g. [GS99] for more details. Let $\Omega_{G}(M)=\left(S \mathfrak{g}^{*} \otimes \Omega(M)\right)^{G}$ be the Cartan double complex of the $G$-manifold $M$. For brevity let us write $\Omega=\Omega(M)$ and $\Omega_{G}=\Omega_{G}(M)$. Elements of $\Omega_{G}$ can be regarded as equivariant polynomial maps from $\mathfrak{g}$ to $\Omega$ and are called equivariant differential forms on $M$. The bigrading on the Cartan complex is defined by $\Omega_{G}^{i j}=\left(S^{i} \mathfrak{g}^{*} \otimes \Omega^{j-i}\right)^{G}$. It is equipped with the vertical differential $1 \otimes d$, which we will abbreviate to $d$, and the horizontal differential $\partial$, which is defined by $\partial \alpha(\xi)=-\iota(\xi) \alpha(\xi)$. Here $\iota(\xi)$ denotes inner product with the vector field on $M$ induced by $\xi \in \mathfrak{g}$. We can regard $\Omega_{G}$ as a single complex with grading $\Omega_{G}^{k}=\bigoplus_{i+j=k} \Omega_{G}^{i j}$ and total differential $d_{G}=d+\partial$, which is called the equivariant exterior derivative. If $d_{G} \alpha=0$, resp. $\alpha=d_{G} \beta$, we call $\alpha$ equivariantly closed, resp. equivariantly exact. The total cohomology ker $d_{G} / \mathrm{im} d_{G}$ is the equivariant de Rham cohomology $H_{G}(M)$. Observe that $\Omega_{G}^{0 j}=\left(\Omega^{G}\right)^{j}$, the space of 
invariant $j$-forms on $M$. Thus the zeroth column of the Cartan complex $\Omega_{G}$ is the invariant de Rham complex $\Omega^{G}$, which is a deformation retract of the ordinary de Rham complex $\Omega$ because $G$ is connected. Hence $H\left(\Omega^{G}\right)=$ $H(\Omega)=H(M)$. The Cartesian projection $\bar{p}: \Omega_{G} \rightarrow \Omega^{G}$, defined by $\bar{p}(\alpha)=$ $\alpha(0)$, is a chain map with respect to the equivariant exterior derivative $d_{G}$ on $\Omega_{G}$ and the ordinary exterior derivative $d$ on $\Omega^{G}$. The Cartan complex is a de Rham model for the homotopy quotient $M_{G}$, which is the total space of the bundle with fibre $M$ associated to the universal $G$-bundle over the classifying space $B_{G}$. The projection $\bar{p}$ models the inclusion of the fibre $M \hookrightarrow M_{G}$.

However, symplectic Hodge theory gives us a fourth differential $1 \otimes \delta$ of bidegree $(0,-1)$, which we will write as $\delta$.

Lemma 3.1. $\partial \delta=-\delta \partial$ and $d_{G} \delta=-\delta d_{G}$.

Proof. Let $\pi$ be the Poisson two-vector defined by the symplectic form. Using Proposition 2.5(i) we find for all $\alpha \in \Omega_{G}$

$$
\begin{aligned}
\partial \delta \alpha(\xi) & =(-\iota(\xi) \iota(\pi) d+\iota(\xi) d \iota(\pi)) \alpha(\xi) \\
& =(\iota(\pi) d \iota(\xi)-d \iota(\pi) \iota(\xi)-\iota(\pi) \mathcal{L}(\xi)+\mathcal{L}(\xi) \iota(\pi)) \alpha(\xi), \\
\delta \partial \alpha(\xi) & =(-\iota(\pi) d \iota(\xi)+d \iota(\pi) \iota(\xi)) \alpha(\xi),
\end{aligned}
$$

so that $(\partial \delta+\delta \partial) \alpha(\xi)=(\mathcal{L}(\xi) \iota(\pi)-\iota(\pi) \mathcal{L}(\xi)) \alpha(\xi)$. Now $\alpha\left(\operatorname{Ad} g^{-1}(\xi)\right)=$ $g^{*} \alpha(\xi)$ for all $g \in G$ and $\xi \in \mathfrak{g}$ and hence $\alpha([\eta, \xi])=\mathcal{L}(\eta) \alpha(\xi)$ for all $\xi$, $\eta \in \mathfrak{g}$. Likewise, since $\pi$ is invariant, $\iota(\pi) \alpha\left(\operatorname{Ad} g^{-1}(\xi)\right)=g^{*} \iota(\pi) \alpha(\xi)$ and so $\iota(\pi) \alpha([\eta, \xi])=\mathcal{L}(\eta) \iota(\pi) \alpha(\xi)$. Hence $\iota(\pi) \mathcal{L}(\xi) \alpha(\xi)=\mathcal{L}(\xi) \iota(\pi) \alpha(\xi)=0$ and $(\partial \delta+\delta \partial) \alpha(\xi)=0$, i.e. $\partial \delta=-\delta \partial$. Since $d \delta=-\delta d$, this implies $d_{G} \delta=$ $(d+\partial) \delta=-\delta(d+\partial)=-\delta d_{G}$.

This implies that $\Omega_{G, \delta}=\operatorname{ker} \delta \cap \Omega_{G}$ is a double subcomplex of $\Omega_{G}$ and that the homology $H\left(\Omega_{G}, \delta\right)$ of $\Omega_{G}$ with respect to $\delta$ is a double complex with differentials induced by $d$ and $\partial$. Thus we have a diagram of morphisms of double complexes

$$
\Omega_{G} \longleftarrow \Omega_{G, \delta} \longrightarrow H\left(\Omega_{G}, \delta\right)
$$

Since $\delta$ does not act on the polynomial part, these morphisms are linear over the invariant polynomials $\left(S \mathfrak{g}^{*}\right)^{G}$. Let us first examine the complex $H\left(\Omega_{G}, \delta\right)$. We need a preliminary result about the action of $\iota(\xi)$ on invariant forms. It follows from the identity $\mathcal{L}(\xi)=d \iota(\xi)+\iota(\xi) d$ that $\iota(\xi): \Omega^{G} \rightarrow \Omega^{G}$ is a chain map (of degree 1) with respect to $d$. Likewise, the identity $\partial \delta+\delta \partial=0$ of Lemma 3.1 applied to the zeroth column of $\Omega_{G}$ shows that $\iota(\xi)$ is a chain map with respect to $\delta$.

Lemma 3.2. Let $\xi \in \mathfrak{g}$ and $\alpha \in \Omega^{G}$. If $\alpha$ is closed, then $\iota(\xi) \alpha$ is exact. If $\alpha$ is coclosed, then $\iota(\xi) \alpha$ is coexact. Equivalently, for every $\xi \in \mathfrak{g}$ the maps

$$
\iota_{d}: H\left(\Omega^{G}, d\right) \rightarrow H\left(\Omega^{G}, d\right), \quad \iota_{\delta}: H\left(\Omega^{G}, \delta\right) \rightarrow H\left(\Omega^{G}, \delta\right)
$$


induced by $\iota(\xi): \Omega^{G} \rightarrow \Omega^{G}$ are zero.

Proof. The fact that $\iota_{\delta}=0$ (which is true without the Lefschetz condition) follows from Proposition 2.5(iii) and the assumption that the $G$-action is Hamiltonian. The assertion $\iota_{d}=0$ is due to Ginzburg [Gin87]. For the reader's convenience we recall the argument. Let $\alpha \in \Omega^{n-k}$ be invariant and closed. Recall that the class $[\alpha] \in H^{n-k}(M)$ is called primitive if $\left[\omega^{k+1} \wedge \alpha\right]=0$, and that strong Lefschetz implies the Lefschetz decomposition $H^{i}(M)=\bigoplus_{j \geq 0}\left[\omega^{j}\right] P^{i-2 j}$, where $P^{i} \subseteq H^{i}(M)$ is the subspace of primitive elements. First consider the case that $[\alpha]$ is primitive. Suppose $\iota_{d}[\alpha] \in$ $H^{n-k+1}(M)$ was nonzero. The identity $\iota(\xi)(\omega \wedge \alpha)=d \phi(\xi) \wedge \alpha+\omega \wedge \iota(\xi) \alpha$ implies that $\iota_{d}$ commutes with multiplication by the symplectic class $[\omega]$. Thus by strong Lefschetz

$$
\iota_{d}\left[\omega^{k+1} \wedge \alpha\right]=[\omega]^{k+1} \wedge \iota_{d}[\alpha] \neq 0,
$$

which contradicts $\left[\omega^{k+1} \wedge \alpha\right]=0$. Therefore $\iota_{d}[\alpha]=0$ for $[\alpha]$ primitive. It now follows from the primitive decomposition that $\iota_{d}[\alpha]=0$ for all $[\alpha]$.

This enables us to show that $H\left(\Omega_{G}, \delta\right)$ is a trivial double complex.

Lemma 3.3. Both differentials on $H\left(\Omega_{G}, \delta\right)$ are zero.

Proof. First note that the (ordinary) $d \delta$-lemma, Theorem 2.8, holds for equivariant forms as well as for ordinary forms. The reason is that $d$ and $\delta$ act on $\Omega_{G}$ as $1 \otimes d$ and $1 \otimes \delta$ and that both operators are $G$-equivariant. Now suppose $\alpha \in \Omega_{G}$ satisfies $\delta \alpha=0$. Then $d \alpha=d \delta \beta$ for some $\beta \in \Omega_{G}$ by the $d \delta$-lemma. Hence $d \alpha=-\delta d \beta$, so the differential on $H\left(\Omega_{G}, \delta\right)$ induced by $d$ is zero. To prove that the other differential is zero we must be more careful about picking a representative of an element of $H\left(\Omega_{G}, \delta\right)$. Observe that

$$
H\left(\Omega_{G}, \delta\right)=\left(S \mathfrak{g}^{*} \otimes H(\Omega, \delta)\right)^{G}=\left(S \mathfrak{g}^{*}\right)^{G} \otimes H(M),
$$

where we used the isomorphism $H(\Omega, \delta) \cong H(M)$ of Theorem 2.9 and the connectedness of $G$ to ensure that $G$ acts trivially on $H(M)$ and $H(\Omega, \delta)$. (It is easy to see that the inclusion $\Omega^{G} \hookrightarrow \Omega$ is a deformation retraction for $\delta$ as well as for $d$.) Choose a basis $\left\{\xi_{q}\right\}$ of $\mathfrak{g}$, let $\left\{x_{q}\right\}$ be the dual basis of $\mathfrak{g}^{*}$ and let $\left\{f_{p}\right\}$ be a basis of the vector space $\left(S \mathfrak{g}^{*}\right)^{G}$ of invariant polynomials. It follows from (3) that an element of $H\left(\Omega_{G}, \delta\right)$ can be represented by an $\alpha \in \Omega_{G}$ with $\delta \alpha=0$ of the form $\alpha=\sum_{p} f_{p} \otimes \alpha_{p}$ for unique $\alpha_{p} \in \Omega^{G}$. Then we have $\delta \alpha_{p}=0$ for all $p$. Using Lemma $3.2\left(\iota_{\delta}=0\right)$ we find $\beta_{p q} \in \Omega^{G}$ such that $\iota\left(\xi_{q}\right) \alpha_{p}=\delta \beta_{p q}$. Thus

$$
\partial \alpha=\sum_{p q} x_{q} f_{p} \otimes \iota\left(\xi_{q}\right) \alpha_{p}=\delta \beta
$$

where $\beta=\sum_{p q} x_{q} f_{p} \otimes \beta_{p q} \in S \mathfrak{g}^{*} \otimes \Omega$. After averaging over $G$ we get $\partial \alpha=\delta \beta$ with $\beta \in \Omega_{G}$, i.e. the differential on $H\left(\Omega_{G}, \delta\right)$ induced by $\partial$ is zero. 
Let $E$ be the spectral sequence of $\Omega_{G}$ relative to the filtration associated to the horizontal grading and $E_{\delta}$ that of $\Omega_{G, \delta}$. The first terms are

$$
\begin{gathered}
E_{1}=\operatorname{ker} d / \operatorname{im} d=\left(S \mathfrak{g}^{*} \otimes H(M)\right)^{G}=\left(S \mathfrak{g}^{*}\right)^{G} \otimes H(M), \\
\left(E_{\delta}\right)_{1}=(\operatorname{ker} d \cap \operatorname{ker} \delta) /(\operatorname{im} d \cap \operatorname{ker} \delta)=\left(S \mathfrak{g}^{*} \otimes H_{\delta}(M)\right)^{G} \\
=\left(S \mathfrak{g}^{*}\right)^{G} \otimes H(M) .
\end{gathered}
$$

Here we used the isomorphism $H_{\delta}(M) \cong H(M)$ of Theorem 2.9 and the connectedness of $G$. By Lemma $3.3 H\left(\Omega_{G}, \delta\right)$ is a trivial complex, so its spectral sequence is constant with trivial differentials at each stage. The two morphisms (2) induce morphisms of spectral sequences

$$
E \longleftarrow E_{\delta} \longrightarrow H\left(\Omega_{G}, \delta\right)
$$

It follows from (3) and (4) that these morphisms are isomorphisms at the first stage. Hence they are isomorphisms at every stage. In particular the three sequences converge to the same limit, so the morphisms (2) induce isomorphisms on total cohomology. In fact, since the spectral sequence for $H\left(\Omega_{G}, \delta\right)$ is constant, so are the spectral sequences $E$ and $E_{\delta}$. This proves the following result, where $H_{G, \delta}(M)$ denotes the total cohomology of $\Omega_{G, \delta}$.

Theorem 3.4 (equivariant formality). The spectral sequences $E$ and $E_{\delta}$ degenerate at the first term. The morphisms (2) induce isomorphisms of $\left(S \mathfrak{g}^{*}\right)^{G}$-modules

$$
H_{G}(M) \stackrel{\cong}{\cong} H_{G, \delta}(M) \stackrel{\cong}{\longrightarrow}\left(S \mathfrak{g}^{*}\right)^{G} \otimes H(M) .
$$

Thus the equivariant de Rham complex of $M$ is formal, i.e. quasi-isomorphic to a complex with zero differential.

The spectral sequence $E$ is isomorphic to the Leray spectral sequence of the fibre bundle $M_{G} \rightarrow B_{G}$ and its degeneracy means that the map induced by $\bar{p}$,

$$
p: H_{G}(M) \rightarrow H(M),
$$

is surjective, in other words that each cohomology class on the fibre $M$ can be extended to an equivariant cohomology class. We assert that there is a natural choice of such an extension. Let

$$
s: H(M) \rightarrow H_{G}(M)
$$

be the composition of the map $H(M) \hookrightarrow\left(S \mathfrak{g}^{*}\right)^{G} \otimes H(M)$ which sends a cohomology class $c$ to $1 \otimes c$ and the isomorphism $\left(S \mathfrak{g}^{*}\right)^{G} \otimes H(M) \rightarrow H_{G}(M)$ given by Theorem 3.4 .

Corollary 3.5. $s$ is a section of $p$. Thus every cohomology class has a canonical equivariant extension. 
Proof. Via the isomorphisms $H(M) \cong H(\Omega, \delta)$ and $H_{G}(M) \cong H\left(\Omega_{G}, \delta\right)$ given by Theorems 2.9 and 3.4 the map $p$ corresponds to the natural projection $\left(S \mathfrak{g}^{*}\right)^{G} \otimes H(\Omega, \delta) \rightarrow H(\Omega, \delta)$ and $s$ corresponds to the inclusion $H(\Omega, \delta) \hookrightarrow\left(S \mathfrak{g}^{*}\right)^{G} \otimes H(\Omega, \delta)$ defined by $c \mapsto 1 \otimes c$. Therefore $p \circ s=1$.

Corollary 3.6. If $\alpha \in \Omega_{G}$ is closed, then $\partial \alpha$ is exact. More generally, let $j \geq 1$ and suppose for $0 \leq i<j$ equivariant forms $\zeta_{i} \in \Omega_{G}$ are given such that $d \zeta_{0}=0$ and $\partial \zeta_{i-1}+d \zeta_{i}=0$ for $1 \leq i<j$. Then there exists $\zeta_{j} \in \Omega_{G}$ such that $\partial \zeta_{j-1}+d \zeta_{j}=0$.

Proof. This is equivalent to the degeneracy of $E$. (Cf. the description of the differentials on $E$ in [BT82, p. 164].)

These results improve on [Kir84, Proposition 5.8] and [Gin87, Theorem 3.3], which state that $E$ degenerates and that $H_{G}(M) \cong S \mathfrak{g}^{*} \otimes H(M)$ noncanonically. However, the cited results hold for arbitrary compact Hamiltonian $G$-manifolds, whereas we have imposed the Lefschetz condition. For completeness we record how in principle one can compute the section $s$. Let $[\alpha] \in H^{k}(M)$. Let us assume, as we may, that the representative $\alpha$ is invariant and harmonic. Then $d \alpha=0$ implies that $\partial \alpha$ is exact by the first statement in Corollary 3.6, and $\delta \alpha=0$ implies that $\partial \alpha$ is coclosed. The $d \delta$-lemma gives $\beta_{1} \in \Omega_{G}^{1 k}$ such that

$$
\partial \alpha=-d \delta \beta_{1} .
$$

This equation shows that $\partial d \delta \beta_{1}=0$, so $d \partial \delta \beta_{1}=-\partial d \delta \beta_{1}=0$. Also $\partial \delta \beta_{1}=$ $-\delta \partial \beta_{1}$ is coexact, so another application of the $d \delta$-lemma yields $\partial \delta \beta_{1}=$ $-d \delta \beta_{2}$ for some $\beta_{2} \in \Omega_{G}^{2, k-1}$. Recursively solving the equation $\partial \delta \beta_{i}=$ $-d \delta \beta_{i+1}$ for $\beta_{i+1}$ in this manner we find a finite chain of $\beta_{i} \in \Omega_{G}^{i, k+1-i}$. Put

$$
\alpha_{G}=\alpha+\sum_{i} \delta \beta_{i} \in \Omega_{G}^{k}
$$

Then $d_{G} \alpha_{G}=\delta \alpha_{G}=0$ and $\bar{p} \alpha_{G}=\alpha$ by construction. Let $\{\alpha\}$, resp. $\left\{\alpha_{G}\right\}$, be the homology class of $\alpha$ in $H(\Omega, \delta)$, resp. of $\alpha_{G}$ in $H\left(\Omega_{G}, \delta\right)=$ $\left(S \mathfrak{g}^{*}\right)^{G} \otimes H(\Omega, \delta)$. Since $\alpha_{G}-\alpha=\sum_{i} \delta \beta_{i}$ is coexact, the element $1 \otimes\{\alpha\} \in$ $\left(S \mathfrak{g}^{*}\right)^{G} \otimes H(\Omega, \delta)$ is equal to $\left\{\alpha_{G}\right\}$. Therefore $s([\alpha])=\left[\alpha_{G}\right]$. (The point of Corollary 3.5 is that the equivariant cohomology class of $\alpha_{G}$ is independent of the choice of the $\beta_{i}$.)

Example 3.7. The equivariant symplectic form $\omega+\phi \in \Omega_{G}^{2}$ is an equivariantly closed extension of the symplectic form, but $\phi$ is not necessarily coexact, so we may not have $s[\omega]=[\omega+\phi]$. Put $\chi_{0}(\xi)=\int_{M} \phi(\xi) \omega^{n} / n$ !. Then $\chi_{0}: \mathfrak{g} \rightarrow \mathbf{R}$ is a character of $\mathfrak{g}$. According to Example 2.4 the shifted moment map $\phi_{0}=\phi-\chi_{0}$ is coexact, so $\omega_{G}=\omega+\phi_{0}$ is the equivariant extension for which $s[\omega]=\left[\omega_{G}\right]$. 
As an aside we point out that $s$ (or any other section of $p$ ) is very far from being a ring homomorphism. Indeed, if $s$ was multiplicative we would have $0=s\left(\omega^{n+1}\right)=\left[\omega_{G}\right]^{n+1}$, i.e. $\left(\omega_{G}\right)^{n+1}=\phi \omega^{n}+\cdots+\phi^{n+1}$ would be equivariantly exact. In particular $\partial \lambda=\phi^{n+1}$ for some $\lambda \in \Omega_{G}^{n, n+1}$, i.e. $-\iota(\xi) \lambda(\xi)=\phi(\xi)^{n+1}$ for all $\xi \in \mathfrak{g}$. The extrema of $\phi(\xi)$ are critical points for the vector field induced by $\xi$, so this equality implies that $\phi(\xi)$ vanishes identically. Thus $s$ is multiplicative only in the uninteresting case of a trivial action.

Example 3.8. As the preceding remark indicates, extending the powers of the symplectic class is not entirely straightforward. As an example we find $s\left([\omega]^{2}\right)$. The moment map $\phi_{0}$ of Example 3.7 is coexact, so $\phi_{0}=\delta \phi_{1}$ for some $\phi_{1} \in \Omega_{G}^{12}$. Then $\frac{1}{2} \phi_{0}^{2}+\partial \phi_{1} \in \Omega_{G}^{22}$, so

$$
\chi_{1}(\xi)=\int_{M}\left(\frac{1}{2} \phi_{0}(\xi)^{2}+\partial \phi_{1}(\xi)\right) \frac{\omega^{n}}{n !}
$$

defines an invariant quadratic polynomial on $\mathfrak{g}$. Moreover, by Example 2.4 $\frac{1}{2} \phi_{0}^{2}+\partial \phi_{1}-\chi_{1}=\delta \phi_{2}$ for some $\phi_{2} \in \Omega_{G}^{23}$. A computation shows that $\omega^{2}-2 \delta\left(\omega \wedge \phi_{1}\right)-\delta \phi_{2}$ is equivariantly closed. Therefore

$$
s\left([\omega]^{2}\right)=\left[\omega^{2}-2 \delta\left(\omega \wedge \phi_{1}\right)-\delta \phi_{2}\right] .
$$

A variation on the construction of equivariant extensions gives us the $d_{G} \delta$ lemma. Call an equivariant form $\alpha$ equivariantly harmonic if it is equivariantly closed $\left(d_{G} \alpha=0\right)$ and coclosed $(\delta \alpha=0)$.

Theorem 3.9 ( $d_{G} \delta$-lemma). Suppose $\alpha \in \Omega_{G}$ is equivariantly harmonic and either equivariantly exact or coexact. Then there exists $\beta \in \Omega_{G}$ such that $\alpha=d_{G} \delta \beta$.

Proof. Let $\alpha \in \Omega_{G}^{k}$ and assume $\alpha=d_{G} \gamma$ and $\delta \alpha=0$. Decomposing $\alpha=$ $\sum_{i} \alpha_{i}$ and $\gamma=\sum_{i} \gamma_{i}$ into homogeneous parts $\alpha_{i} \in \Omega_{G}^{i, k-i}$ and $\gamma_{i} \in \Omega_{G}^{i, k-i-1}$ we have

$$
\begin{aligned}
\delta \alpha_{i} & =0 \\
\alpha_{i} & =\partial \gamma_{i-1}+d \gamma_{i}
\end{aligned}
$$

for $i \geq 0$, where we put $\gamma_{-1}=0$. We need to solve $\alpha=d_{G} \delta \beta$ for $\beta \in \Omega_{G}^{k}$, i.e. we must find $\beta_{i} \in \Omega_{G}^{i, k-i}$ such that

$$
\alpha_{i}=\partial \delta \beta_{i-1}+d \delta \beta_{i}
$$

for $i \geq 0$, where again we put $\beta_{-1}=0$. The zeroth equation $\left(\ddagger_{0}\right)$ amounts to $\alpha_{0}=d \delta \beta_{0}$, which is soluble by the $d \delta$-lemma because the integrability conditions $\left(*_{0}\right)$ and $\left(\dagger_{0}\right)$ say that $\alpha_{0}$ is coclosed and exact. Now let $j \geq 1$ 
and assume that $\left(\ddagger_{i}\right)$ has been solved for $i<j$. Write $\zeta_{i}=\gamma_{i}-\delta \beta_{i}$ for $-1 \leq i<j$. Then $\zeta_{-1}=0$. Also

$$
d \zeta_{i}=d\left(\gamma_{i}-\delta \beta_{i}\right)=-\partial \gamma_{i-1}+\partial \delta \beta_{i-1}=-\partial \zeta_{i-1}
$$

for $0 \leq i<j$ by $\left(\dagger_{i}\right)$ and $\left(\ddagger_{i}\right)$. Hence by Corollary 3.6 there exists $\zeta_{j}$ such that $\partial \zeta_{j-1}+d \zeta_{j}=0$. Now rewrite $\left(\ddagger_{j}\right)$ as

$$
d \delta \beta_{j}=\alpha_{j}-\partial \delta \beta_{j-1}
$$

and observe that by $\left(*_{j}\right)$ the right-hand side of this equation is coclosed. Using $\left(\dagger_{j}\right)$ we rewrite the right-hand side as

$$
\alpha_{j}-\partial \delta \beta_{j-1}=\partial \gamma_{j-1}+d \gamma_{j}-\partial \delta \beta_{j-1}=\partial \zeta_{j-1}+d \gamma_{j}=d\left(-\zeta_{j}+\gamma_{j}\right),
$$

which is exact. So $\left(\ddagger_{j}\right)$ is soluble by the $d \delta$-lemma. By induction $\left(\ddagger_{i}\right)$ is soluble for all $i \geq 0$, so we find $\beta$ with $\alpha=d_{G} \delta \beta$. The second half of the $d_{G} \delta$-lemma follows from the first: assume $d_{G} \alpha=0$ and $\alpha$ coexact. Then the homology class of $\alpha$ in $H\left(\Omega_{G}, \delta\right)$ is zero, so by Theorem 3.4 the cohomology class of $\alpha$ in $H_{G, \delta}(M)$ is zero, i.e. $\alpha$ is equivariantly exact. Hence $\alpha=d_{G} \delta \beta$ for some $\beta$.

Let $\Omega_{G \text {,per }}^{i j}=\Omega_{G}^{j-i}$ be the periodic equivariant de Rham complex of $M$, equipped with the horizontal differential $\delta$ and the vertical differential $d_{G}$. The $d_{G} \delta$-lemma has as an immediate consequence the following equivariant analogue of [Bry88, Theorem 2.3.1].

Corollary 3.10. The spectral sequences of $\Omega_{G \text {,per }}$ with respect to the horizontal and vertical filtrations both degenerate at the first term.

See [Bry88, Section 2.3] for the definition of degeneration for a double complex that is zero below the diagonal $i=j$. (But note Brylinski's opposite conventions for the periodic complex.) As in loc. cit., the degeneracy with respect to the vertical filtration is probably true even if $M$ does not satisfy the Lefschetz condition.

\section{References}

[Bry88] J.-L. Brylinski, A differential complex for Poisson manifolds, J. Differential Geom. 28 (1988), no. 1, 93-114. MR 89m:58006

[BT82] R. Bott and L. Tu, Differential forms in algebraic topology, Springer-Verlag, New York, 1982. MR 83i:57016

[Del68] P. Deligne, Théorème de Lefschetz et critères de dégénérescence de suites spectrales, Inst. Hautes Études Sci. Publ. Math. 35 (1968), 259-278. MR 39 \#5582

[Gin87] V. A. Ginzburg, Equivariant cohomology and Kähler geometry, Funktsional. Anal. i Prilozhen. 21 (1987), no. 4, 19-34, 96, English translation in Functional Anal. Appl. 21 (1987), no. 4, 271-283. MR 89b:58013

[GS99] V. Guillemin and S. Sternberg, Supersymmetry and equivariant de Rham theory, Mathematics Past and Present, Springer-Verlag, Berlin, 1999. MR 2001i:53140 
[Gui01] V. Guillemin, Symplectic Hodge theory and the $d \delta$-lemma, preprint, Massachusetts Institute of Technology, 2001.

[Kir84] F. Kirwan, Cohomology of quotients in symplectic and algebraic geometry, Mathematical Notes, vol. 31, Princeton University Press, Princeton, N.J., 1984. MR 86i: 58050

[Kos85] J.-L. Koszul, Crochet de Schouten-Nijenhuis et cohomologie, Élie Cartan et les mathématiques d'aujourd'hui (Lyon, 1984), Astérisque, numéro hors série, Société Mathématique de France, 1985, pp. 257-271. MR 88m:17013

[Mat95] O. Mathieu, Harmonic cohomology classes of symplectic manifolds, Comment. Math. Helv. 70 (1995), no. 1, 1-9. MR 96e:58004

[Mer98] S. Merkulov, Formality of canonical symplectic complexes and Frobenius manifolds, Internat. Math. Res. Notices 14 (1998), 727-733. MR 99j:58078

[Yan96] D. Yan, Hodge structure on symplectic manifolds, Adv. Math. 120 (1996), no. 1, 143-154. MR 97e:58004

Department of Mathematics, Cornell University, IthacA, New York 148537901, USA

E-mail address: linyi@math.cornell.edu

Department of Mathematics, Cornell University, Ithaca, New York 148537901, USA

E-mail address: sjamaar@math.cornell.edu

R. Sjamaar was partially supported by NSF Grant DMS-0071625. 